\title{
CLDN3 expression and function in pregnancy-induced hypertension
}

\author{
AIXIN ZHAO, YUNFANG QI and KUN LIU \\ Department of Obstetrics, Laiwu Maternal and Child Health Hospital, Laiwu, Shandong 271199, P.R. China
}

Received July 9, 2018; Accepted June 20, 2019

DOI: $10.3892 /$ etm.2020.9084

\begin{abstract}
This aim of the present study was to investigate the expression and function of claudin 3 (CLDN3) in pregnancy-induced hypertension. The mRNA expression levels of CLDN3 in the placental tissue and peripheral blood of patients with pregnancy-induced hypertension were measured using reverse transcription-quantitative PCR. Human trophoblast HTR8/SVneo cells overexpressing CLDN3 were generated using a lentiviral vector. Cell Counting kit-8 (CCK-8) assay, flow cytometry, Transwell chamber assays, confocal laser scanning microscopy and western blot analysis were performed to detect cell proliferation, invasion, migration and apoptosis, in addition to matrix metalloproteinase (MMP) expression and ERK1/2 phosphorylation. The mRNA expression levels of CLDN3 were significantly reduced in the placental tissues and peripheral blood samples of patients with pregnancy-induced hypertension compared with healthy pregnant controls. CLDN3 overexpression significantly increased HTR8/SVneo cell proliferation, invasion and migration whilst reducing apoptosis. HTR8/SVneo cells overexpressing CLDN3 also exhibited increased myofiber levels, increased MMP-2 and MMP-9 expression and increased ERK1/2 signaling activity. CLDN3 downregulation may be associated with the pathogenesis of pregnancy-induced hypertension. In conclusion, CLDN3 promotes the proliferative and invasive capabilities of human trophoblast cells, with the underlying mechanisms possibly involving upregulation of MMP expression via the ERK1/2 signaling pathway.
\end{abstract}

\section{Introduction}

Pregnancy-induced hypertension is a common disease observed during the gestational period. If not treated in time,

Correspondence to: Dr Aixin Zhao, Department of Obstetrics, Laiwu Maternal and Child Health Hospital, 12 West Street, Laiwu, Shandong 271199, P.R. China

E-mail: aixinzhaoai@sina.com

Key words: pregnancy-induced hypertension, tight junction proteins, claudin 3, matrix metalloproteinases, extracellular signal-regulated kinases $1 / 2$ pregnancy-induced hypertension may cause significant harm to the mother and fetus $(1,2)$. Recent clinical studies have shown that maternal mortality as a result of pregnancy-induced hypertension to be 4.2-10 million worldwide as of 2017, accounting for $\sim 9 \%$ of all maternal deaths and $2.2 \%$ of perinatal child mortality (3). Thus, this disease poses a serious threat to maternal and child health, and represents one of the main causes for the death of pregnant women and neonates (4). The clinical symptoms of pregnancy-induced hypertension mainly include transient hypertension and proteinuria in pregnant women, which normally disappear following delivery (5). At present, pregnancy-induced hypertension is generally divided into five types: Gestational hypertension, pre-eclampsia (mild and severe), eclampsia, chronic hypertension complicated by pre-eclampsia and chronic hypertension combined with pregnancy (6). Pregnant women with severe pregnancy-induced hypertension may suffer from hemolysis, thrombocytopenia, liver and kidney dysfunction, pulmonary edema and visual disturbances (5). Risk factors of the disease include obesity, pre-pregnancy hypertension, diabetes and old age (7). The causes for pregnancy-induced hypertension remain to be elucidated; however, they may be associated with changes in the immune system such as histocompatibility antigenassociated immunological abnormalities (8). In addition, genetic susceptibility may also be involved in the pathogenesis of this disease $(9,10)$.

Trophoblast cells are one of the components of the maternal placental architecture, involved in the regulation of placental microenvironment remodeling, implantation of embryos and normal fetal development (11). Previous studies have shown that trophectoderm cells can differentiate into two types of trophoblast cells at the early stages of blastocyst implantation, namely cytotrophoblast cells and syncytiotrophoblast cells $(12,13)$. Cytotrophoblast cells can fuse with syncytiotrophoblast cells, which differentiate into extravillous trophoblasts (EVTs). Some EVTs infiltrate the deeper layers of the endometrium, and are known as interstitial trophoblast cells (14), whilst others invade the maternal uterine spiral artery, and are called endovascular trophoblast cells (15). Trophoblast cells exhibit similar migratory capacities to tumor cells, which are closely related in biological function (16). Studies have shown that EVTs migrate from the placental villi and invade the endometrium and maternal spiral arteries, where they participate in uterine artery revascularization and regulate placental blood flow and immune responses (17). 
Downregulation of the invasive ability of trophoblast cells can result in defects in uterine spiral artery remodeling and placental insufficiency, leading to pregnancy-induced hypertension, eclampsia and miscarriage (18). Therefore, it is of great clinical significance to study changes in trophoblast cell invasion and the associated mechanism in the pathogenesis of pregnancy-induced hypertension.

Tight junction proteins are important in the maintenance of cell-to-cell connections, which serve important roles in cell polarity and the formation of cellular barriers such as the intestinal epithelial barrier $(19,20)$. In particular, claudins (CLDNs) are members of the tight junction protein family that serve important roles in the formation of tight junctions. In total, 24 CLDNs have been identified (21). In recent years, studies have demonstrated that CLDN3 is abnormally expressed in a number of tumor tissues, including hepatocellular carcinoma and breast cancer $(22,23)$ and closely associated with the invasion and metastasis of tumor cells $(24,25)$. Notably, when tumor cells metastasize, the tight junctions between cells must be destroyed, and CLDN3 is an important component of these junctions (26). During trophoblast cell invasion, the breaking of tight junctions is also an important prerequisite for detachment (27). However, the function of CLDN3 in this process remain unclear. Therefore, in the present study, the regulatory role of CLDN3 in the invasive abilities of trophoblast cells was investigated, on tissue and cellular levels.

\section{Materials and methods}

Study subjects and sample collection. A total of 51 pregnant women with hypertension, including 25 patients diagnosed with pregnancy-induced hypertension, 11 patients with mild pre-eclampsia and 15 patients with severe pre-eclampsia, and 30 normal pregnant women were included in this study, all of whom were admitted to Laiwu Maternal and Child Health Hospital (Laiwu, China) for delivery from December 2016 to December 2017. In these patients, the pregnancy hypertension was defined as: i) $\mathrm{BP} \geq 140 / 90 \mathrm{mmHg}$ during pregnancy, which returned to normal within 12 weeks after delivery; ii) urine protein (-); and iii) cases that may be associated with upper abdominal discomfort or thrombocytopenia. Mild pre-eclampsia was defined as: i) $\mathrm{BP} \geq 140 / 90 \mathrm{mmHg}$ appeared during pregnancy, which returned to normal within 12 weeks after delivery; ii) urine protein (-); and iii) cases that may be associated with upper abdominal discomfort or thrombocytopenia. Severe pre-eclampsia was defined as maternal convulsions that cannot be explained by other reasons. None of the pregnant women had previous pregnancies or suffered from hypertension, diabetes or underlying diseases, including liver, kidney and autoimmune diseases, prior to the present pregnancy. All these patients received cesarean section due to pregnancy-induced hypertension, abnormal fetal position or other social factors. There were no significant differences in age, gestational period or neonatal weight between the pregnancy-induced hypertension and control group (mean age of patients with pregnancy-induced hypertension, $29.5 \pm 1.08$ years; mean gestational period, $38 \pm 2.08$ weeks; mean neonatal weight, $7.6 \pm 2.3 \mathrm{~kg}$. Mean age of patients with mild-pre-eclampsia, 33.1 \pm 1.08 years; gestational period, $39 \pm 3.08$ weeks; neonatal weight, $7.1 \pm 1.3 \mathrm{~kg}$. Mean age of patients with severe pre-eclampsia, $31.45 \pm 1.08$ years; gestational period, $40 \pm 2.60$ weeks; neonatal weight, $7.7 \pm 1.7 \mathrm{~kg}$ ). Prior written informed consent was obtained from every patient and the study was approved by the ethics review board of Laiwu Maternal and Child Health Hospital. In total $10 \mathrm{ml}$ peripheral blood sample was obtained from each subject and the collection of placental tissues was performed $10 \mathrm{~min}$ after the placenta was delivered, with a $2 \times 2 \times 2 \mathrm{~cm}$ sample of tissue dissected from the central area (avoiding infarction and calcification) of the maternal placenta. The tissue was washed with saline immediately after collection, and then stored at $-80^{\circ} \mathrm{C}$.

Reverse transcription-quantitative PCR (RT-qPCR). Total RNA was extracted from the placental tissue and peripheral blood samples using TRIzol ${ }^{\circledR}$ (Thermo Fisher Scientific, Inc.) according to the manufacturer's protocol. RNA concentrations were quantified using the NanoDrop method. Reverse transcription was performed with $0.5 \mu \mathrm{g}$ RNA to obtain cDNA using BeyoRT ${ }^{\mathrm{TM}}$ cDNA First Chain Synthesis kit (cat. no. D7166; Beyotime Institute of Biotechnology). Subsequent qPCR was performed using BeyoFast ${ }^{\mathrm{TM}}$ SYBR Green qPCR Mix (cat. no. 7260; Beyotime Institute of Biotechnology) in a StepOnePlus ${ }^{\mathrm{TM}}$ Real-Time PCR instrument. The following primer sequences were used: CLDN3 forward, 5'-GCCACCAAGGTCGTCTACTC-3' and reverse, 5'-CCTGCGTCTGTCCCTTAGAC-3' and GAPDH forward, 5'-CGGAGTCAACGGATTTGGTCGTAT-3' and reverse, 5'-AGCCTTCTCCATGGTGGTGAAGAC-3'. The total $20 \mu \mathrm{l}$ PCR mixture consisted of $10 \mu \mathrm{l}$ RT-qPCR-Mix, $0.5 \mu \mathrm{l}$ each primer, $2 \mu \mathrm{l} \mathrm{cDNA}$ and $7 \mu \mathrm{l} \mathrm{ddH}_{2} \mathrm{O}$. The thermocycling conditions were as follows: Initial denaturation at $95^{\circ} \mathrm{C}$ for $10 \mathrm{~min}$; followed by 40 cycles of $95^{\circ} \mathrm{C}$ for $1 \mathrm{~min}$ and $60^{\circ} \mathrm{C}$ for $1 \mathrm{~min}$. Target gene expression levels were calculated using the $2^{-\Delta \Delta \mathrm{Cq}}$ method (28). GAPDH was used as internal reference.

Human trophoblast cell culture. Normal human trophoblast HTR8/SVneo cells were purchased from American Type Culture Collection. Cells were cultured using RPMI-1640 medium (Gibco; Thermo Fisher Scientific, Inc.) containing $10 \%$ fetal bovine serum (FBS; Gibco; Thermo Fisher Scientific, Inc.) and supplemented with $100 \mathrm{U} / \mathrm{ml}$ penicillin and $100 \mathrm{U} / \mathrm{ml}$ streptomycin, in a humidified atmosphere at $37^{\circ} \mathrm{C}$ containing $5 \% \mathrm{CO}_{2}$. When cell confluence reached $90 \%$, the cells were passaged using $0.5 \%$ trypsin.

Construction and transfection of lentivirus vector for CLDN3. The lentiviral Lv-GFP-CLDN3 vector was constructed by Hanbio Biotechnology Co., Ltd., with the titer of $1 \times 10^{8}$ pfu. HTR8/SVneo cells were first seeded into 24-well plates at a density of $1 \times 10^{5}$ cells/well and cultured with RPMI-1640 medium containing $10 \%$ FBS. When $70 \%$ confluence was achieved, the HTR8/SVneo cells were transfected with either empty vector or Lv-GFP-CLDN3 at a multiplicity of infectionof 20. The medium was changed to fresh RPMI-1640 medium containing 10\% FBS after $6 \mathrm{~h}$, and the cells were cultured at $37^{\circ} \mathrm{C}$ under $5 \% \mathrm{CO}_{2}$ for a further $48 \mathrm{~h}$.

Cell counting kit (CCK)-8 assay. CCK-8 assay (Beyotime Institute of Biotechnology) was performed to measure the 
viability of the HTR8/SVneo cells. Briefly, at $48 \mathrm{~h}$ after transfection, the cells were seeded into 96 -well plates at $2 \times 10^{3}$ cells/well. A total of $200 \mu \mathrm{l}$ RPMI-1640 complete medium containing $10 \% \mathrm{FBS}$ and $100 \mathrm{U} / \mathrm{ml}$ penicillin and streptomycin was added and the cells were then incubated at $37^{\circ} \mathrm{C}$ under $5 \% \mathrm{CO}_{2}$. Following cell adhesion, at $0,24,48$ and $72 \mathrm{~h}$, the cells were incubated with $20 \mu \mathrm{l} \mathrm{CCK}-8$ reaction solution for $30 \mathrm{~min}$. The optical density values at $490 \mathrm{~nm}$ were then obtained for each well using a microplate reader, which were used to produce a cell viability curve.

Flow cytometry. Flow cytometry was performed to measure HTR8/SVneo cell proliferation and apoptosis. For cell proliferation, the cells were collected and washed with PBS. Following fixation with $4 \%$ formaldehyde for $10 \mathrm{~min}$ at room temperature, the cells were centrifuged at $1,000 \mathrm{xg}$ for $5 \mathrm{~min}$ at room temperature. The cells were treated with $0.5 \%$ Triton $\mathrm{X}-100$ at room temperature for $5 \mathrm{~min}$ and then incubated with BD Cytofix/Cytoperm ${ }^{\mathrm{TM}}$ Plus reagent (cat. no. 555028; BD Biosciences) at room temperature in the dark for $15 \mathrm{~min}$. Fluorescence was detected and the percentage of $\mathrm{Ki}-67$ calculated from it using the BD FACSVerse ${ }^{\mathrm{TM}}$ flow cytometer (BD Biosciences) and the FlowJo ${ }^{\mathrm{TM}} \mathrm{VX} 10$ software (FlowJo LLC).

For apoptosis detection, $48 \mathrm{~h}$ after transfection, $1 \times 10^{6} \mathrm{HTR} 8 / \mathrm{SVneo}$ cells were collected and cultured with a shaker for $2 \mathrm{~h}$ at a speed of $200 \mathrm{rpm}$. After washing twice with ice-cold PBS, the cells were labeled using the BD Pharmingen $^{\text {TM }}$ FITC Annexin V Apoptosis Detection Kit I (cat. no. 556547; BD Biosciences), according to the manufacturer's protocol. Flow cytometry was conducted to detect the fluorescence, from which apoptosis rate was calculated.

For Ki-67 detection, the cells were collected and treated with BD Cytofix/Cytoperm ${ }^{\mathrm{TM}}$ Plus reagent (cat. no. 555028; BD Biosciences) according to the manufacturer's protocols. The cells were then incubated with $\mathrm{PE}-\mathrm{Cy}^{\mathrm{TM}} 7$-conjugatedmouse anti-Ki-67 antibody (cat. no. 561283; BD Biosciences) in the dark at room temperature for $15 \mathrm{~min}$, followed by detection of the fluorescence with flow cytometry.

Transwell chamber assay. The infiltration ability of the cells was detected using Transwell chamber assays. Matrigel ${ }^{\circledR}$ solution was diluted in serum-free RPMI-1640 medium (v:v, 1:3), evenly smeared onto the upper chamber and incubated at $37^{\circ} \mathrm{C}$ for $60 \mathrm{~min}$. The HTR8/SVneo cells were then seeded onto the upper chamber at a density of $1 \times 10^{5}$ cells/well and cultured in $200 \mu 1$ serum-free RPMI-1640 medium while $500 \mu 1$ RPMI-1640 medium containing $20 \%$ FBS was present in the lower chamber. After $24 \mathrm{~h}$, the cells were fixed with $4 \%$ formaldehyde at room temperature for $10 \mathrm{~min}$ and subjected to Giemsa staining at room temperature for $2 \mathrm{~min}$. Following rinsing for $2 \mathrm{~min}$, the cells were air-dried. HTR8/SVneo cells that infiltrated to the lower chamber were observed and counted under a light microscope, from a total of five random fields of view under high magnification (x200). For the measurements of cell migration, identical procedures were performed as described aforementioned using Transwell chambers that were not coated with Matrigel.

Confocal laser scanning microscopy (CLSM). The cytoskeleton was imaged using CLSM. After transfection, when $80 \%$ confluence was reached, HTR8/SVneo cells were fixed with $4 \%$ formaldehyde at room temperature for $10 \mathrm{~min}$. After washing with PBS, the cells were permeabilized using $0.5 \%$ Triton X-100 at room temperature for $5 \mathrm{~min}$ and incubated with $200 \mu 1100 \mathrm{nmol} / \mathrm{l}$ Rhodamine Phalloidin at room temperature in the dark for $30 \mathrm{~min}$. The cytoskeleton was then observed using a confocal microscope (no. of fields taken, 5; magnification, x400; model SP8; Leica Microsystems GmbH).

Western blot analysis. At $48 \mathrm{~h}$ after transfection, the cells were lysed using RIPA buffer (Beyotime Institute of Biotechnology) supplemented with PMSF (100 mM) on ice. The protein concentration was determined using Bicinchoninic Acid assay (Beyotime Institute of Biotechnology). Then, $20 \mu \mathrm{g}$ sample was separated by $10 \%$ SDS-PAGE, and then transferred onto PVDF membranes. After blocking with $50 \mathrm{~g} / 1$ fat-free milk diluted in $0.1 \%$ TBST at room temperature for $1 \mathrm{~h}$, the membranes were incubated withrabbit anti-human anti-CLDN3 (1:1,000 dilution; cat. no. 83609), rabbit anti-human anti-matrix metalloprotease (MMP)-2 (1:1,000 dilution; cat no. 40994), rabbit anti-human anti-MMP-9 (1:1,000 dilution; cat no. 13667), rabbit antihuman anti-ERK1/2 (1:1,000 dilution; cat no. 5013), mouse anti-human anti-p-ERK1/2 (1:1,000 dilution; cat. no. 9106) or rabbit anti-human anti-GAPDH (1:5,000 dilution; cat no. 5174) primary antibodies (all from Cell Signaling Technology, Inc.) at $4^{\circ} \mathrm{C}$ overnight. After washing with TBST, the membranes were incubated with horseradish peroxidase-conjugated goat anti-rabbit (cat. no. A0208) or goat anti-mouse (cat. no. A0216) secondary antibody (both 1:4,000 dilution; Beyotime Institute of Biotechnology) at room temperature for $1 \mathrm{~h}$. Protein bands were visualized using the ECL method (Pierce; Thermo Fisher Scientific, Inc.) and analyzed using the Quantity one V4.6.7 software (Bio-Rad Laboratories, Inc.).

Statistical analysis. Data are presented as the mean \pm SD. SPSS 20.0 software (IBM Corp.) was used for statistical analysis. Pairwise comparisons were performed using the Student's t-test, whereas one-way ANOVA followed by Dunnett's test was used for multiple group comparisons. $\mathrm{P}<0.05$ was considered to indicate a statistically significant difference.

\section{Results}

Expression of CLDN3 in placental tissues from patients with pregnancy-induced hypertension. To investigate the expression of CLDN3 in the placenta of the pregnant patients with and without hypertension, RT-qPCR was performed. Compared with the healthy control group, the expression levels of CLDN3 mRNA in the placental tissue were significantly reduced in the pregnant patients with hypertension $(\mathrm{P}<0.01$; Fig. 1A). In addition, relative CLDN3 mRNA expression levels in the placental tissues from the severe pre-eclampsia, mild pre-eclampsia and pregnancy-induced hypertension groups were significantly reduced compared with the healthy control group $(\mathrm{P}<0.05$; Fig. 1A). In the peripheral blood, compared with the control group, the relative CLDN3 mRNA expression levels in the pregnancy-induced hypertension, mild pre-eclampsia and severe pre-eclampsia groups were significantly lower $(\mathrm{P}<0.05$; Fig. 1B). These results suggest that at the onset of pregnancy-induced hypertension, CLDN3 expression 

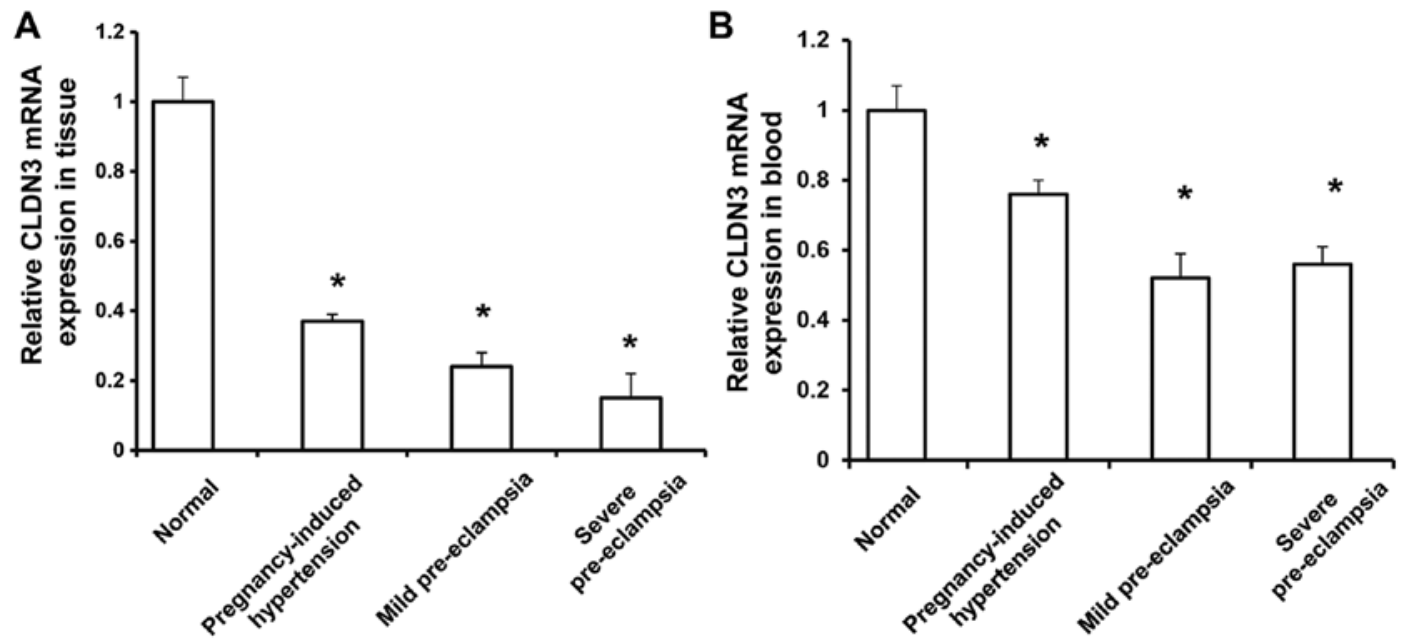

Figure 1. Expression levels of CLDN3 mRNA in the placental tissues and peripheral blood of patients. The mRNA expression levels of CLDN3 in (A) placental tissues and (B) peripheral blood of normal control, pregnancy-induced hypertension, mild pre-eclampsia and severe mild pre-eclampsia groups were measured using reverse transcription quantitative PCR. Experiments were performed in triplicates. "P<0.05 vs. normal. CLDN3, claudin 3.

A
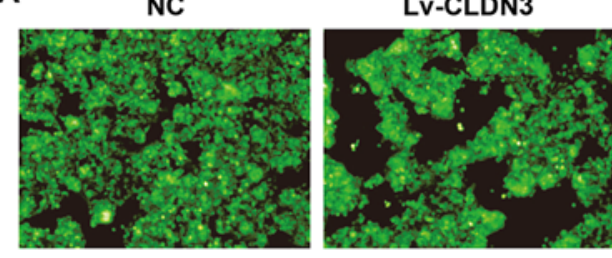

C

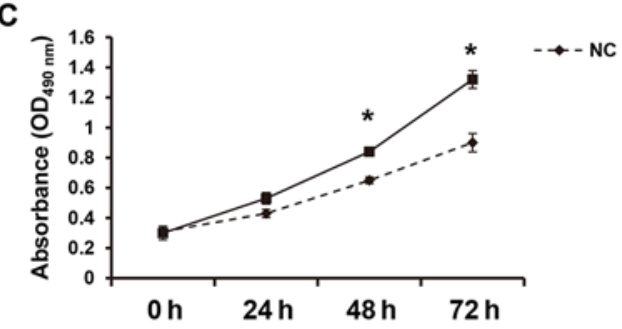

B
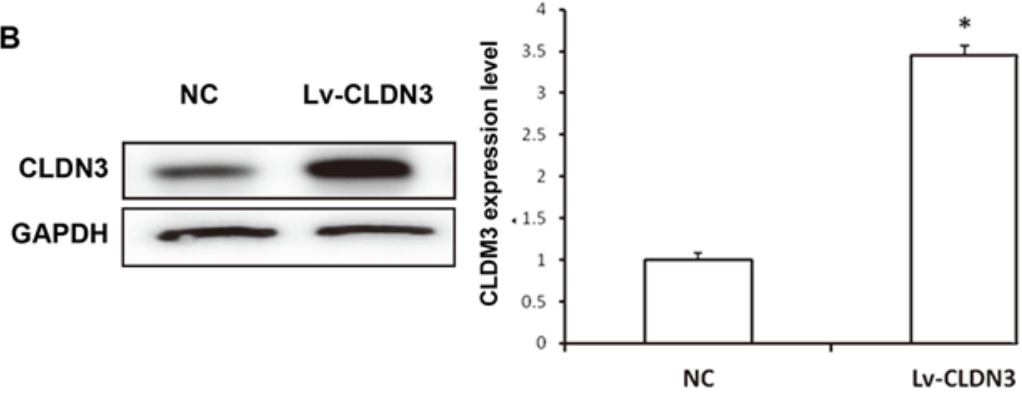

D
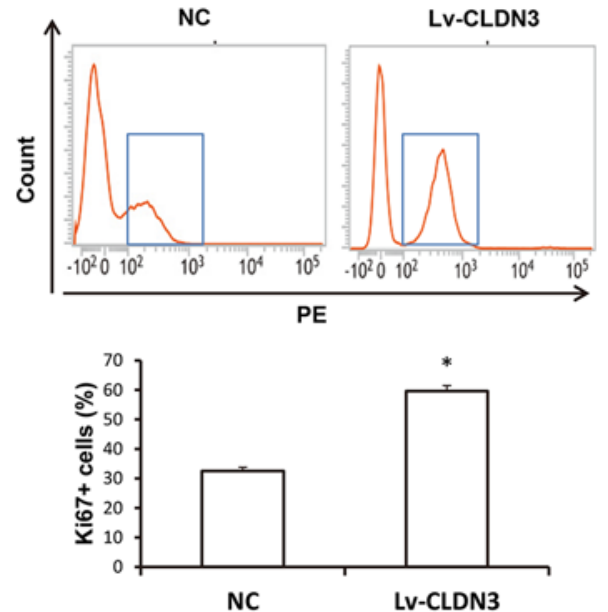

Figure 2. Effects of CLDN3 overexpression on the proliferation of HTR8/SVneo cells. (A) After transfection with Lv-GFP-CLDN3, GFP fluorescence was detected using confocal laser scanning microscopy (magnification, x200). (B) CLDN3 overexpression in HTR8/SVneo cells was verified using western blot analysis. (C and D) Following transfection, cell viability was determined using (C) Cell Counting kit-8 assay and (D) proliferation was assessed via the quantification of Ki-67 using flow cytometry. Experiments were performed in triplicates. "P<0.05 vs. NC. CLDN3, claudin 3; GFP, green fluorescent protein; NC, cells transfected with empty vector; OD, optical density; SSC, side scatter; PE, phycoerythrin.

was reduced in placental tissues, and may be associated with disease pathogenesis.

CLDN3 overexpression enhances of HTR8/SVneocell viability. The effects of CLDN3 on the viability of HTR8/SVneo cells were next investigated. At $48 \mathrm{~h}$ after transfection with Lv-GFP-CLDN3, GFP fluorescence was distributed evenly in the cytoplasm of the HTR8/SVneo cells (Fig. 2A). Western blot analysis confirmed that CLDN3 expression was elevated in HTR8/SVneo cells after transfection with Lv-GFP-CLDN3 compared with those in the empty vector-transfected control $(\mathrm{P}<0.05$; Fig. 2B). The viability of the transfected cells was measured using a CCK- 8 assay. Compared with the control group, CLDN3 overexpression significantly increased 
A
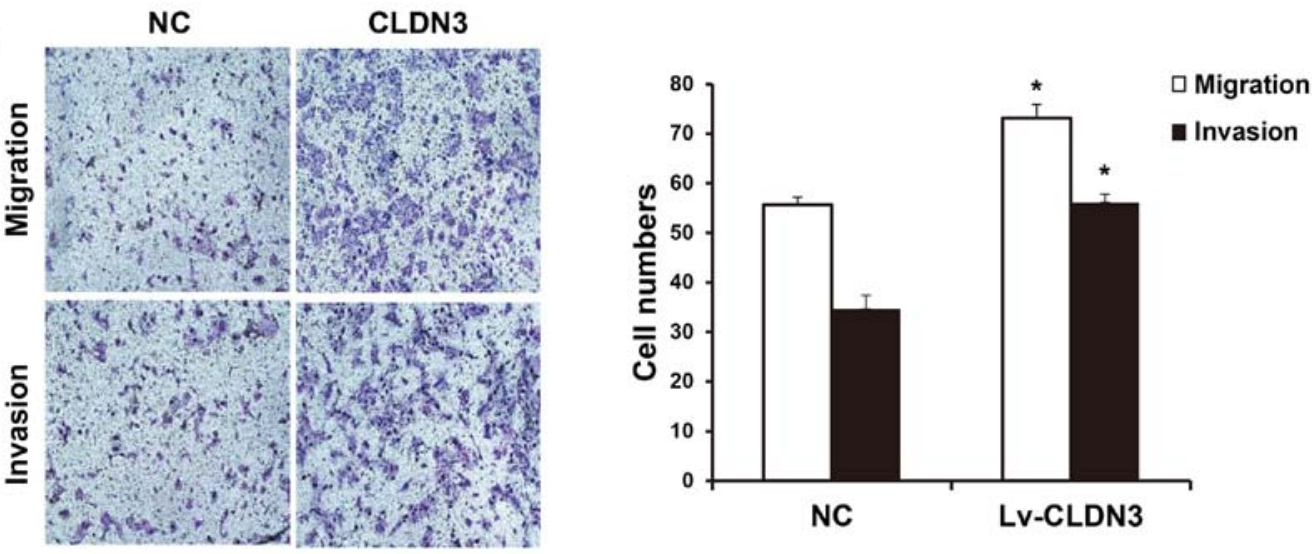

B
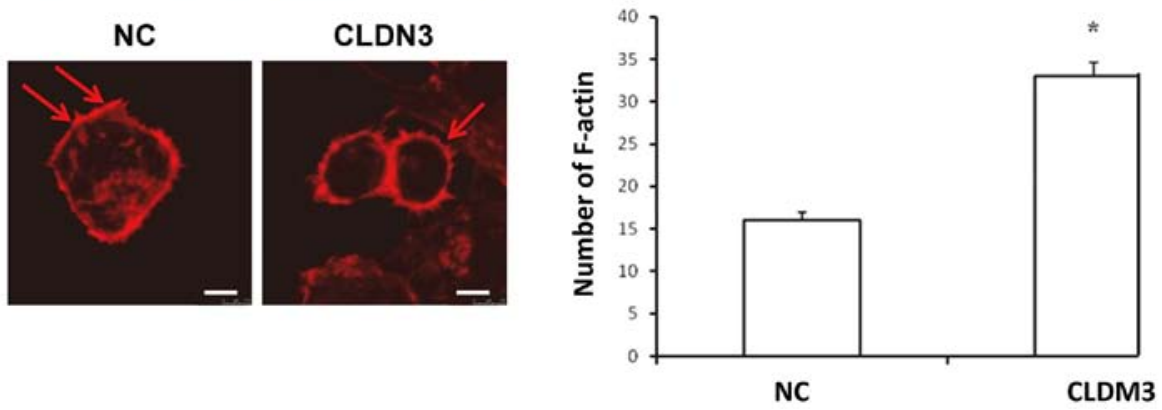

Figure 3. Effects of CLDN3 overexpression on HTR8/SVneo cell invasion and migration. (A) The invasive and migratory capabilities of transfected HTR8/SVneo cells were measured using Transwell chamber assays. (B) The F-actin cytoskeleton (red fluorescence) was imaged using confocal laser scanning microscopy. Scale bar, $2 \mu \mathrm{m} .{ }^{*} \mathrm{P}<0.05$ vs. NC. CLDN3, claudin 3; NC, cells transfected with empty vector.
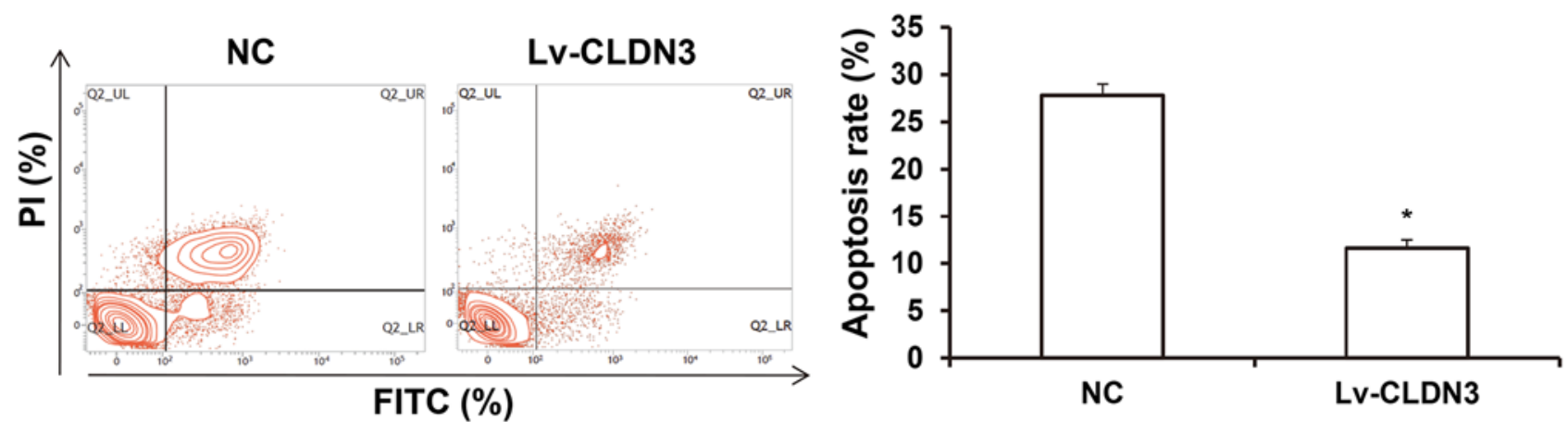

Figure 4. Effects of CLDN3 overexpression on the apoptosis of HTR/SVneo cells. The apoptosis of transfected HTR/SVneo cells was detected using flow cytometry and the apoptotic rates were calculated accordingly. Experiments were performed in triplicates. " $\mathrm{P}<0.05$ vs. NC. CLDN3, claudin 3 ; FITC, fluorescein isothiocyanate; PI, propidium iodide. $\mathrm{NC}$, cells transfected with empty vector.

HTR8/SVneo cell viability at 24,48 and $72 \mathrm{~h}$ after transfection $(\mathrm{P}<0.05$; Fig. $2 \mathrm{C})$. Consistent results were observed using flow cytometry, which indicated that the overexpression of CLDN3 significantly upregulated Ki-67 expression in HTR8/SVneo cells $(\mathrm{P}<0.05$; Fig. 2D). These results indicate that CLDN3 overexpression significantly promoted HTR8/SVneo cell proliferation.

CLDN3 overexpression promotes the invasive and migratory abilities of HTR8/SVneo cells. To investigate the effect of CLDN3 on the invasive and migratory abilities of HTR8/SVneo cells, Transwell chamber assays were performed. Compared with the control group, CLDN3 overexpression significantly promoted the migratory and invasive capabilities of HTR8/SVneo cells ( $\mathrm{P}<0.05$; Fig. 3A). In addition, CLSM results showed that in HTR8/SVneo cells overexpressing CLDN3, the number of F-actin myofibers was significantly increased, indicating increased migratory ability $(\mathrm{P}<0.05$; Fig. 3B). These results suggest that CLDN3 overexpression promotes HTR8/SVneo cell migration and invasion.

Effects of CLDN3 overexpression on HTR8/SVneo cell apoptosis. Apoptosis is also an important factor affecting the migratory and invasive abilities of HTR8/SVneo cells (29). To investigate the effects of CLDN3 on apoptosis in HTR8/SVneo cells, flow cytometry was performed. Compared with the control group, the overexpression of CLDN3 significantly reduced HTR8/SVneo cell apoptosis $(\mathrm{P}<0.05$; Fig. 4). These results suggest that CLDN3 overexpression can suppress apoptosis in HTR8/SVneo cells. 

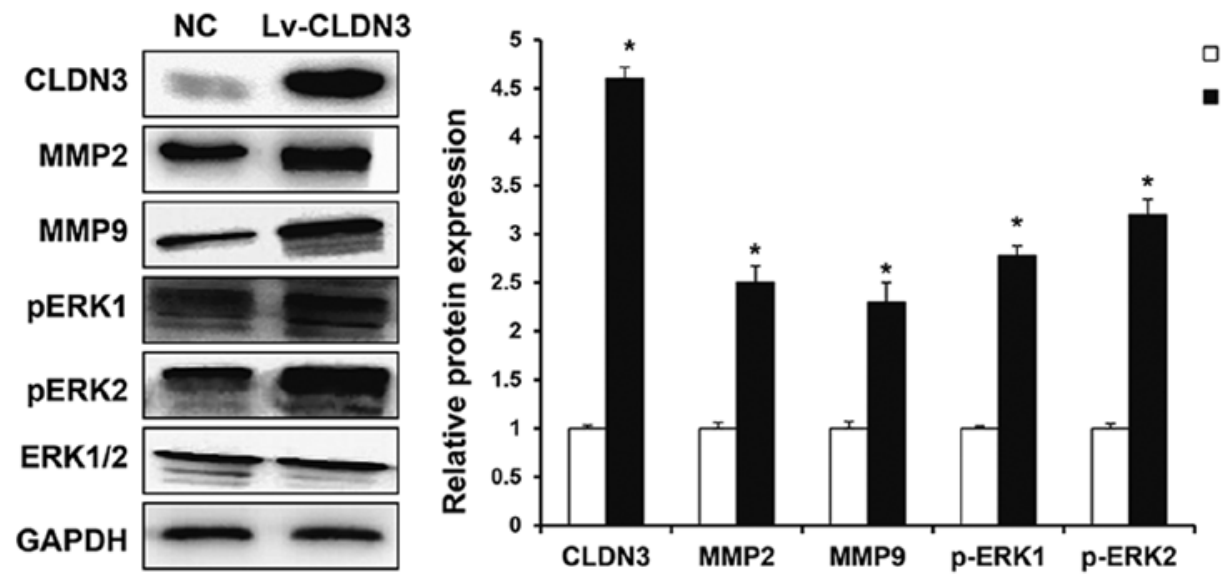

Figure 5. Effects of CLDN3 on MMP expression and ERK phosphorylation in HTR8/SVneo cells. Following transfection, the expression levels of MMP-2 and MMP-9 proteins, in addition to ERK1/2 phosphorylation were detected using western blot analysis. Experiments were performed in triplicates. "P<0.05 vs. NC. CLDN3, claudin 3; MMP, matrix metalloproteinase; NC, cells transfected with empty vector.

Effects of CLDN3 on the ERK1/2 signaling pathway and MMP expression in HTR8/SVneo cells. The ERK1/2/MMP pathway serves an important role in the cell migration and is regulated by tight junction proteins (6). To investigate the effects of CLDN3 on the ERK1/2 signaling pathway (30) in addition to MMP-2 and MMP-9 expression in HTR8/SVneo cells, western blot analysis was performed following CLDN3 overexpression. The overexpression of CLDN3 significantly elevated the expression levels of MMP-2 and MMP-9 in HTR8/SVneo cells. ERK1/2 phosphorylation was also significantly increased following CLDN3 overexpression (Fig. 5). These results suggest that CLDN3 can significantly affect the expression of MMPs and ERK activation in HTR8/SVneo cells.

\section{Discussion}

Pregnancy-induced hypertension is a common complication in obstetrics (31). Clinical manifestations of pregnancy-induced hypertension include proteinuria and fetal growth restriction after 20 weeks of gestation (32). At present, the pathogenesis of this disease remains poorly characterized (33). A previous study has demonstrated that in most women with pregnancy-induced hypertension, the symptoms of hypertension typically disappear following the delivery of placenta, suggesting that the placenta may serve an important role in the development of pregnancy-induced hypertension (34). Trophoblast cells are important for the structure and function of placenta, where the tight junction proteins serve important roles in maintaining the cellular polarity and integrity of cell barriers. However, whether or not the tight junction proteins regulate the biological function of trophoblast cells remains unclear. The present study demonstrated that the expression levels of the tight junction protein CLDN3 were significantly downregulated in the placental tissues of patients with pregnancy-induced hypertension compared with healthy pregnant controls. Supporting this, in vitro experiments using human trophoblast cell line HTR8/SVneo cells showed that CLDN3 overexpression promoted cell proliferation and invasion, possibly by activating the ERK1/2/MMP-2/MMP-9 signaling pathway.
The placenta is the location where gas and nutrient exchange occurs and where metabolic products are eliminated between the mother and the fetus. As part of the main placental structure, invasion of trophoblast cells into the endometrium is an important physiological process in the formation of the placenta $(35,36)$. Studies have shown that extravillous trophoblast cells invade into the uterine decidua and myometrial spiral artery, where they gradually replace the endothelial cell layer and part of the smooth muscle tissue in the maternal spiral arteries, resulting in thickened blood vessels to provide sufficient blood supply to the fetus $(37,38)$. Trophoblast cell invasion is a key factor to this process, and factors hindering this physiological process may lead to placental dysplasia, pregnancy-induced hypertension and eclampsia (39). In particular, one of the pathological changes in the placenta during pre-eclampsia is the shallow and limited invasion of trophoblast cells into the uterine spiral artery (40).

Tight junction proteins, of which the CLDN family of proteins is an important example, serve important roles in the maintenance of cell barriers and polarity. In recent years, a large number of studies have shown that CLDNs are closely associated with tumor cell invasion and metastasis (41). Indeed, CLDN2 has been reported to promote the self-renewal of colon cancer stem cells (42), whereas the downregulation of CLDN12 expression by interleukin (IL)-18 has been demonstrated to activate the p38 pathway to promote the invasion and metastasis of breast cancer in another recent study (43). CLDN3 is an important member of the CLDN family. Studies have confirmed the involvement of CLDN3 in the regulation of tumor cell invasion and metastasis through multiple signaling pathways. Ahmad et al (44) showed that the loss of CLDN3 expression activated the IL-6/glycoprotein 130/STAT3 pathway to promote the malignant transformation of colon cancer. In lung squamous cell carcinoma, CLDN3 could inhibit the invasion and metastasis of lung cancer cells (45). However, it remains to be elucidated whether CLDN3 is involved in the regulation of human trophoblast cell invasion. Results from the present study showed that the mRNA expression levels of CLDN3 in the placental tissues and peripheral blood of patients with pregnancy-induced hypertension were significantly reduced compared with those in healthy controls, 
indicating a negative association with disease pathogenesis. The in vitro experiments of the present study indicated that the overexpression of CLDN3 promoted HTR8/SVneocell proliferation, migration and invasion. During invasion, human trophoblast cells would detach from adjacent cells or the extracellular matrix. Normal cells undergo apoptosis to maintain the stability of the tissue environment, whereas tumor cells or other migratory cells may suppress apoptosis to promote their migration. In the present study, CLDN3 overexpression significantly inhibited the apoptosis of HTR8/SVneo cells. These observations suggest that CLDN3 promotes the proliferation, in addition to the invasive and migratory abilities of HTR8/SVneo cells in vitro. The present study demonstrated changes in CLDM3 expression in human tissues and cells, and also detected similar changes in the levels of CLDN3 in the peripheral blood. From these observations it could be hypothesized that the placental tissue released CLDM3 into the circulation; however, there isno direct evidence that the placenta is the sole source of CLDM3.

It has previously been found that human trophoblast cells secrete MMPs to degrade the extracellular matrix during invasion (46). MMP-2 and MMP-9 are the main enzymes involved in the degradation of the basal membrane of the uterus (47). It has been reported that MMP-2 and MMP-9 could assist in the invasion of human trophoblast cells, and MMP-2 and MMP-9 can degrade extracellular matrix, making cells more susceptible to metastasis and infiltration $(48,49)$. In the present study, results from western blot analysis showed that CLDN3 overexpression significantly elevated the expression levels of MMP-2 and MMP-9 in human trophoblast cells. Regulation of MMPs involves a number of different factors, including cytokine profiles, signaling pathway activation and MMP-inhibiting factors. A previous study found that both the expression and activity of MMP-9 are regulated by the ERK1/2 signaling pathway (50). Indeed, the present study showed that the overexpression of CLDN3 significantly elevated the phosphorylation levels of ERK1 and ERK2 in HTR8/SVneo cells, suggesting that CLDN3 activates the ERK1/2 signaling pathway.

However, in the present study, although the expression levels of CLDN3 in the placental tissue and the effects on the biological function of trophoblast cells were investigated, only a single trophoblast cell line was used, and further in-depth studies on primary trophoblast cells are required to confirm the regulatory effects of CLDN3.

In conclusion, the present study showed that CLDN3 can promote human trophoblast cell proliferation, migration and invasion, with the underlying mechanism possibly involving the upregulation of MMP-2 and MMP-9 expression levels via the ERK1/2 signaling pathway. These findings suggest that downregulation of CLDN3 may be associated with the pathogenesis of pregnancy-induced hypertension, which may serve to assist in the design of therapeutic interventions for the treatment of this disease in the clinic.

\section{Acknowledgements}

The authors thank Director Ailan Wang from the Laiwu Maternal and Child Health Hospital (Shandong, China) for kind assistance in the study design, experiment performance, data analysis and manuscript preparation.

\section{Funding}

No funding was received.

\section{Availability of data and materials}

All data generated or analyzed during this study are included in this published article.

\section{Authors' contributions}

AZ, YQ and KL contributed to the study design, experiment performance, data collection and analysis, and manuscript preparation. All authors read and approved the final manuscript.

\section{Ethics approval and consent to participate}

The study was approved by the ethics review board of Laiwu Maternal and Child Health Hospital (Shandong, China). Prior written informed consent was obtained from every patient.

\section{Patient consent for publication}

Not applicable.

\section{Competing interests}

The authors declare that they have no competing interests.

\section{References}

1. Ali AM, Alobaid A, Malhis TN and Khattab AF: Effect of vitamin D3 supplementation in pregnancy on risk of pre-eclampsia-randomized controlled trial. Clin Nutr 38: 557-563, 2018.

2. Leikin JB: Renal complications during pregnancy: In the hypertension spectrum. Dis Mon 65: 24, 2018.

3. Ray JG, Diamond P, Singh G and Bell CM: Brief overview of maternal triglycerides as a risk factor for pre-eclampsia. BJOG 113: 379-386, 2006.

4. Tripathy K, Chawla R, Mutha V and Selvan H: Spontaneous suprachoroidalhaemorrhage with exudative retinal detachment in pregnancy-induced hypertension. BMJ Case Rep 9: 223907, 2018.

5. Shawkat E, Mistry H, Chmiel C, Webster L, Chappell L, Johnstone ED and Myers JE: The effect of labetalol and nifedipine MR on blood pressure in women with chronic hypertension in pregnancy. Pregnancy Hypertens 11: 92-98, 2018.

6. Rhodes CA, Beevers DG and Churchill D: A randomized trial of ambulatory blood pressure monitoring versus clinical blood pressure measurement in the management of hypertension in pregnancy. A feasibility study. Pregnancy Hypertens 11: 142-144, 2018.

7. Braga F, Ferraro S, Borille S and Panteghini M: Biological variation of two serum markers for preeclampsia prediction. Clin Chem Lab Med 28: e27-e28, 2019.

8. Chatterjee P, Chiasson VL, Seerangan G, De Guzman E, Milad M, Bounds KR, Gasheva O, Tobin RP, Hatahet M, Kopriva S, et al: Depletion of MHC class II invariant chain peptide or $\gamma-\delta$ T-cells ameliorates experimental preeclampsia. Clin Sci (Lond) 131: 2047-2058, 2017.

9. Webster LM, Gill C, Seed PT, Bramham K, Wiesender C, Nelson-Piercy C, Myers JE and Chappell LC: Chronic hypertension in pregnancy: The impact of ethnicity and superimposed preeclampsia on placental, endothelial and renal biomarkers. Am J Physiol Regul Integr Comp Physiol 1: R36-R47, 2018. 
10. Sava RI, March KL and Pepine CJ: Hypertension in pregnancy: Taking cues from pathophysiology for clinical practice. Clin Cardiol 41: 220-227, 2018.

11. Gingrich J, Pu Y, Roberts J, Karthikraj R, Kannan K, Ehrhardt R and Veiga-Lopez A: Gestational bisphenol S impairs placental endocrine function and the fusogenic trophoblast signaling pathway. Arch Toxicol 92: 1861-1876, 2018.

12. Chang TA, Bondarenko GI, Gerami-Naini B, Drenzek JG, Durning M, Garthwaite MA, Schmidt JK and Golos TG: Trophoblast differentiation, invasion and hormone secretion in a three-dimensional in vitro implantation model with rhesus monkey embryos. Reprod Biol Endocrinol 16: 24, 2018.

13. Lee CQE, Turco MY, Gardner L, Simons BD, Hemberger M and Moffett A: Integrin $\alpha 2$ marks a niche of trophoblast progenitor cells in first trimester human placenta. Development 16: 145, 2018.

14. Qin X, Liang Y, Guo Y, Liu X, Zeng W, Wu F, Lin Y and Zhang Y: Eukaryotic initiation factor $5 \mathrm{~A}$ and $\mathrm{Ca}^{2+} /$ calmodulin-dependent protein kinase 1D modulate trophoblast cell function. Am J Reprod Immunol 80: e12845, 2018.

15. Xu J,Xia Y,Zhang H, GuoH,Feng K and Zhang C: Overexpression of long non-coding RNA H19 promotes invasion and autophagy via the PI3K/AKT/mTOR pathways in trophoblast cells. Biomed Pharmacother 101: 691-697, 2018.

16. Midic U, Goheen B, Vincent KA, VandeVoort CA and Latham KE: Changes in gene expression following long-term in vitro exposure of macacamulatta trophoblast stem cells to biologically relevant levels of endocrine disruptors. Reprod Toxicol 77: 154-165, 2018.

17. Zheng J, Wang $\mathrm{H}$ and Zhou W: Modulatory effects of trophoblast-secreted CXCL12 on the migration and invasion of human first-trimester decidual epithelial cells are mediated by CXCR4 rather than CXCR7. Reprod Biol Endocrinol 16: 17, 2018.

18. HudonThibeault AA, Vaillancourt $C$ and Sanderson JT: Profile of CYP19A1 mRNA expression and aromatase activity during syncytialization of primary human villous trophoblast cells at term. Biochimie 148: 12-17, 2018.

19. Tian H, Miao J, Zhang F, Xiong F, Zhu F, Li J, Wang X, Chen S, Chen J, Huang $\mathrm{N}$ and Wang Y: Non-histone nuclear protein HMGN2 differently regulates the urothelium barrier function by altering expression of antimicrobial peptides and tight junction protein genes in UPEC J96-infected bladder epithelial cell monolayer. Acta Biochim Pol 65: 93-100, 2018.

20. Matsuoka H, Tamura A, Kinehara M, Shima A, Uda A, Tahara H and Michihara A: Levels of tight junction protein CLDND1 are regulated by microRNA-124 in the cerebellum of stroke-prone spontaneously hypertensive rats. Biochem Biophys Res Commun 498: 817-823, 2018.

21. Altunbulakli C, Costa R, Lan F, Zhang N, Akdis M, Bachert C and Akdis CA: Staphylococcus aureus enhances the tight junction barrier integrity in healthy nasal tissue, but not in nasal polyps. J Allergy Clin Immunol 142: 665-668, 2018.

22. Kim SO, Choi BT, Choi IW, Cheong J, Kim GY, Kwon TK, Kim ND and Choi YH: Anti-Invasive activity of histone deacetylase inhibitors via the induction of Egr-1 and the modulation of tight junction-related proteins in human hepatocarcinoma cells. BMB Rep 42: 655-660, 2009.

23. Jaaskelainen A, Soini Y, Jukkola-Vuorinen A, Auvinen P, Haapasaari KM and Karihtala P: High-Level cytoplasmic claudin 3 expression is an independent predictor of poor survival in triple-negative breast cancer. BMC Cancer 18: 223, 2018.

24. Piontek A, Rossa J, Protze J, Wolburg H, Hempel C, Günzel D, Krause G and Piontek J: Polar and charged extracellular residues conserved among barrier-forming claudins contribute to tight junction strand formation. Ann N Y Acad Sci 1397: 143-156, 2017.

25. Torres-Martinez AC, Gallardo-Vera JF, Lara-Holguin AN, Montano LF and Rendon-Huerta EP: Claudin-6 enhances cell invasiveness through claudin-1 in AGS human adenocarcinoma gastric cancer cells. Exp Cell Res 350: 226-235, 2017.

26. Akizuki R, Shimobaba S, Matsunaga T, Endo S and Ikari A: Claudin-5, -7, and -18 suppress proliferation mediated by inhibition of phosphorylation of akt in human lung squamous cell carcinoma. Biochim Biophys Acta 1864: 293-302, 2017.

27. Deng Q, Yin N, Chen Y, Shan N, Liu X and Qi H: Downregulated $\mathrm{N}$-acetylglucosaminyltransferase III is involved in attenuating trophoblast migration and invasion under hypoxia-reoxygenation condition. J Matern Fetal Neonatal Med 32: 1-7, 2018.
28. Livak KJ and Schmittgen TD: Analysis of relative gene expression data using real-time quantitative PCR and the 2(-Delta Delta C(T)) method. Methods 25: 402-408, 2001.

29. Li X, Ma XL, Tian FJ, Wu F, Zhang J, Zeng WH, Lin Y and Zhang Y: Downregulation of CCNA2 disturbs trophoblast migration, proliferation, and apoptosis during the pathogenesis of recurrent miscarriage. Am J Reprod Immunol 82: e13144, 2019.

30. Mishra JS, Te Riele GM, Qi QR, Lechuga TJ, Gopalakrishnan K, Chen DB and Kumar S: Estrogen receptor- $\beta$ mediates estradiol-induced pregnancy-specific uterine artery endothelial cell angiotensin type-2 receptor expression. Hypertension 74: 967-974, 2019.

31. Rashidi F and Sate H: Pregnancy outcome in a pregnant patient with idiopathic pulmonary arterial hypertension: A case report and review of the literature. J Med Case Rep 12: $31,2018$.

32. Liao QP, Xu Q and Yan JY: SHH expression in placental tissues and trophoblast cell oxidative stress injury during preeclampsia. Eur Rev Med Pharmacol Sci 23: 6026-6034, 2019.

33. Chen S, Li N, Mei Z, Ye R, Li Z, Liu J and Serdula MK: Micronutrient supplementation during pregnancy and the risk of pregnancy-induced hypertension: A randomized clinical trial. Clin Nutr 38: 146-151, 2018.

34. Tateishi A, Ohira S, Yamamoto Y and Kanno H: Histopathological findings of pregnancy-induced hypertension: Histopathology of early-onset type reflects two-stage disorder theory. Virchows Arch 472: 635-642, 2018.

35. Yang C, Lim W, Bazer FW and Song G: Butyl paraben promotes apoptosis in human trophoblast cells through increased oxidative stress-induced endoplasmic reticulum stress. Environ Toxicol 33: 436-445, 2018.

36. Nikolaou S, Hadjikypri X, Ioannou G, Elia A and Georgiades P: Functional and phenotypic distinction of the first two trophoblast subdivisions and identification of the border between them during early postimplantation: A prerequisite for understanding early patterning during placentogenesis. Biochem Biophys Res Commun 496: 64-69, 2018.

37. Trinh QD, Pham NTK, Takada K, Komine-Aizawa S and Hayakawa S: Myelin oligodendrocyte glycoprotein-independent rubella infection of keratinocytes and resistance of first-trimester trophoblast cells to rubella virus in vitro. Viruses 10: E23, 2018.

38. Xu P, Zhao Y, Liu K, Lin S, Liu X, Wang M, Yang P, Tian T, Zhu YY and Dai Z: Prognostic role and clinical significance of trophoblast cell surface antigen 2 in various carcinomas. Cancer Manag Res 9: 821-837, 2017.

39. Cheng JC, Yi Y, Chang HM and Leung PCK: TGF- $\beta 1$ upregulates cadherin-11 expression through snail: A potential mechanism for human trophoblast cell differentiation. Cell Signal 43: 55-61, 2018

40. Chang CW, Wakeland AK and Parast MM: Trophoblast lineage specification, differentiation and their regulation by oxygen tension. J Endocrinol 236: R43-R56, 2018.

41. Hashimoto Y, Fukasawa M, Kuniyasu H, Yagi K and Kondoh M: Claudin-Targeted drug development using anti-claudin monoclonal antibodies to treat hepatitis and cancer. Ann N Y Acad Sci 1397: 5-16, 2017.

42. Paquet-Fifield S, Koh SL, Cheng L, Beyit LM, Shembrey C, Mølck C, Behrenbruch C, Papin M, Gironella M, Guelfi S, et al: Tight junction protein claudin-2 promotes self-renewal of human colorectal cancer stem-like cells. Cancer Res 78: 2925-2938, 2018.

43. Yang Y, Cheon S, Jung MK, Song SB, Kim D, Kim HJ, Park H, Bang SI and Cho D: Interleukin-18 enhances breast cancer cell migration via downregulation of claudin-12 and induction of the p38 MAPK pathway. Biochem Biophys Res Commun 459: 379-386, 2015.

44. Ahmad R, Kumar B, Chen Z, Chen X, Müller D, Lele SM, Washington MK, Batra SK, Dhawan P and Singh AB: Loss of claudin-3 expression induces IL6/gp130/Stat3 signaling to promote colon cancer malignancy by hyperactivating wnt $/ \beta$-catenin signaling. Oncogene 36: 6592-6604, 2017.

45. Che J, Yue D, Zhang B, Zhang H, Huo Y, Gao L, Zhen H, Yang Y and Cao B: Claudin-3 inhibits lung squamous cell carcinoma cell epithelial-mesenchymal transition and invasion via suppression of the wnt/ $\beta$-catenin signaling pathway. Int J Med Sci 15: 339-351, 2018.

46. Chaudhary P, Babu GS, Sobti RC and Gupta SK: HGF regulate HTR-8/SVneo trophoblastic cells migration/invasion under hypoxic conditions through increased HIF-1 $\alpha$ expression via MAPK and PI3K pathways. J Cell Commun Signal 13: 503-521, 2019. 
47. Liu Y, Shan N, Yuan Y, Tan B,He C, Tong C and Qi H: Knockdown of activated Cdc42-associated kinase inhibits human extravillous trophoblast migration and invasion and decreases protein expression of pho-akt and matrix metalloproteinase. J Matern Fetal Neonatal Med 33: 1-9, 2018.

48. Zhong T, Chen J, Ling Y, Yang B, Xie X, Yu D, Zhang D, Ouyang $\mathbf{J}$ and Kuang H: Down-Regulation of neuropathy target esterase in preeclampsia placenta inhibits human trophoblast cell invasion via modulating MMP-9 levels. Cell Physiol Biochem 45: 1013-1022, 2018.
49. Zong L, Wei X, Gou W, Huang P and Lv Y: Zinc improves learning and memory abilities of fetal growth restriction rats and promotes trophoblast cell invasion and migration via enhancing STAT3-MMP-2/9 axis activity. Oncotarget 8: 115190-115201, 2017.

50. Fu Y, Wei J, Dai X and Ye Y: Increased NDRG1 expression attenuate trophoblast invasion through ERK/MMP-9 pathway in preeclampsia. Placenta 51: 76-81, 2017.

This work is licensed under a Creative Commons Attribution-NonCommercial-NoDerivatives 4.0 International (CC BY-NC-ND 4.0) License. 